\title{
The "-omics" contributions to the understanding of mycotoxin production under diverse environmental conditions
}

\author{
Esther Garcia-Cela, Carol Verheecke-Vaessen, Naresh Magan and Angel Medina* \\ Applied Mycology Group, Environment and AgriFood Theme, Cranfield University, \\ Cranfield Beds MK43 OAL, UK \\ Corresponding author: \\ Angel Medina, Applied Mycology Group, Cranfield Soil and AgriFood Institute, School of \\ Water, Energy and Environment, Vincent Building (52a), Cranfield University, Cranfield, \\ Bedford MK43 OAL, U.K., Tel: +44 (0) $1234-750111$ ext.5045; Email: \\ a.medinavaya@cranfield.ac.uk
}

- EGC and CVV have contributed equally to the writing and development of this work.

\begin{abstract}
Extreme environmental changes and fluctuations mainly driven by climate change will have a profound effect on natural food contaminants. Among these contaminants mycotoxins will be very important due the high adaptability of the producing fungal genera to the forecasted conditions. The availability of modern, high through-put omic techniques, including genomics, transcriptomics, metagenomics, proteomics and metabolomics has facilitated a rapid expansion of data on the biology of mycotoxigenic fungi. This has facilitated a significant increase in our knowledge of the biological, biochemical and biophysical molecular processes regulating the production of mycotoxins, and the adaptation of these fungi to environmental stresses.
\end{abstract}

In this paper we highlight recent advances where -omics approaches have been used and where they have contributed to the knowledge on how mycotoxigenic fungi adapt to diverse interacting environmental conditions and their relationship with phenotypic toxin production. We also highlight potential future directions where these approaches can be effectively utilised for the development of minimisation strategies in the context of expected climate change scenarios and the food security agenda.

\section{Introduction}

When examining different natural food contaminants, especially under extreme environmental events, is there potential for changes in the levels of contamination of staple commodities with mycotoxins [1]. The key fungal genera responsible for production of toxic secondary metabolites in food and feedstuff are Aspergillus, Penicillium, Fusarium and Alternaria. These filamentous fungi exhibit tremendous physiological plasticity which provides them with the capacity for adaptation and 
enables them to colonize food commodities over a range of environmental conditions. Because of their ecological ruderal characteristics, they are able to produce the necessary enzymes and secondary metabolites to provide them with advantages, ecophysioloically, to successfully colonize a range of different food matrices and contaminate the edible parts with mycotoxins [2-4]. Thus, a better understanding of the tolerance and adaptation to diverse environmental conditions and the triggers and cues at a genetic and translational level would be beneficial for a better understanding of mycotoxin production would allow the development of more effective minimisation and control strategies.

The availability of modern, holistic and high through-put -omic techniques, including genomics, transcriptomics, proteomics and metabolomics has facilitated a rapid expansion of data on the biology of these mycotoxigenic fungi (Figure 1). By combining these data sets with advanced computational methods including analyses of a range of genome sequencing projects focused on fungal plant pathogens has had a positive impact on the current state of knowledge available [5]. This has facilitated a significant increase in our knowledge of the biological, biochemical and biophysical molecular processes regulating the production of mycotoxins, especially of Aspergillus flavus and Fusarium species, such as F.graminearum and F.verticillioides [6,7].There have been some studies examining the relationship between interacting conditions of water availability (water activity, $a_{w}$ ) and temperature on expression of gene clusters involved in type A trichothecenes, fumonisins and aflatoxins using microarray analyses which has demonstrated a correlation between growth, biosynthetic gene cluster expression and environmental conditions (see [8]). In most other cases, -omics related studies have investigated genomics or transcriptomics only under one set of environmental condition, often those optimum for growth. However, such conditions are often not related to optimum conditions for secondary metabolite production or indicative of impacts of environmental stress on gene functioning and transcriptomic changes. Recent studies on the transcriptome of $A$. flavus in relation to $a_{w} \times$ temperature stress has suggested significant impacts on different gene clusters, including those for aflatoxins and cyclopiazonic acid production [8-10].

This paper highlights recent advances where -omics approaches have been used, where this has contributed to the knowledge on how mycotoxigenic fungi adapt to diverse interacting environmental conditions and their relationship with phenotypic toxin production. We also highlight potential future directions where these approaches can be effectively utilised for the development of minimisation strategies in the context of expected climate change scenarios and the food security agenda. Although divided in sections, these different approaches can not be considered in isolation and there are multiple links between each other.

\section{Genomics}

The availability of accurate genomic datasets is critical to consider any further transcriptomic level analysis. Regarding mycotoxigenic fungi, since the first genome was published 13 years ago (http://www.aspergillusflavus.org/), more than 30 mycotoxigenic fungal genomes are today openly available (http://www.aspgd.org/, http://genomea.asm.org/). The recent breakthrough involved the genome of Aspergillus westerdijkiae [11] to decipher Ochratoxin A biosynthesis. With genomes 
being available, epigenomic studies have been increasing in the last few years. Regarding $A$. flavus, methylation regulators such as $R m t A, D m t A$ are described as epigenetic factors involved in fungal development and mycotoxin production $[12,13]$. Another example is the impact of chromatin remodelling on FUM1, FUM21 and FUM8 expression in fumonisin production by $F$. verticillioides [14].

\section{Transcriptomics}

A significant number of studies examining the impact of interacting environmental factors on secondary metabolite production were focused on the use of microarrays. Indeed, for a range of mycotoxins a microarray with different sub-arrays have been exploited to enable a better understanding the environmental related stress responses of the gene clusters involved. Examples are shown in the recent reviews by Medina et al., (2015) [7] and Magan \& Medina, (2016) [8]. Complimentary studies using RTqPCR for a better temporal understanding of the relative gene expression of key regulatory and structural genes involved in mycotoxin production for $A$. flavus, $F$. verticillioides and $P$. verrucosum have been done. This has included studies on genes for aflatoxin production (afIR/afIS; afID) [15]; fumonisins (FUM1; FUM21) [16]; ochratoxin A (otaPKS) [17]. However, these studies were focused on a few genes only and did not give a more global understanding of primary and secondary metabolism which is needed to decipher the mechanisms involved. Recently, by using RNAseq it has been possible to provide new insights into the molecular ecology of $A$. flavus and how this might relate to aflatoxins and other secondary metabolite clusters.

The initial transcriptomic studies of $A$. flavus colonisation of maize revealed that the adaptation to temperature conditions $\left(30^{\circ} \mathrm{C}, 37^{\circ} \mathrm{C}\right)$ significantly impacted 1,153 out of the 13,487 genes. At $30^{\circ} \mathrm{C}$, the most highly up-expressed genes belonged to the aflatoxin biosynthesis cluster [18]. More recently, Medina et al. (2017) added a complementary condition by including the interacting effect of $a_{w}(0.91,0.99)$. This showed that 4,307 and 702 genes were affected by a decreasing the water stress at $30^{\circ} \mathrm{C}$ and $37^{\circ} \mathrm{C}$, respectively. Among those, the reduction of $a_{w}$ led to a downexpression in the aflatoxin cluster of up to 7.8 fold [19]. A recent complementary study examined the effect of forecasted climate change environmental conditions which included temperature ( 30 vs $\left.37^{\circ} \mathrm{C}\right)$, aw stress ( 0.99 vs 0.90$)$ and $\mathrm{CO}_{2}$ levels (350 vs. 650 ppmv and $1000 \mathrm{ppmv}$ ). The increase in $\mathrm{CO}_{2}$ mediated the response to temperature and $a_{w}$ allowing for an increase in the aflatoxin B1 accumulation under stress conditions. For example, gene expression was down-regulated for the aflatoxin cluster at $30^{\circ} \mathrm{C}, 0.99$ when increasing the $\mathrm{CO}_{2}$ input [20].

These transcriptomic approaches using RNAseq should provide the foundation starting point for building up datasets to better understand the interaction between primary and secondary metabolism. Further deciphering of the pathways triggered under different interacting environmental factors requires a meta-analysis of transcriptome behaviour by using enhanced bio-informatics tools for the understanding of the different mycotoxin-omes, especially in relation to environmental fluxes. 


\section{Metagenomics -Mycobiome}

The high-throughput sequencing techniques provide a powerful tool to assess the impact of the microenvironment in crops on the "Mycobiome". Recent publications have highlighted the usefulness of those tools [21-23]. Xing et al. (2016) examined peanuts stored for 90 days at $20-30^{\circ} \mathrm{C}$ and relative humidities of 70,75 and $80 \%$. Sequencing analysis revealed an overall decrease of fungal operational taxonomic units (OTU) throughout storage and an increase in $A$. flavus relative abundance throughout the storage period, independent of the conditions tested [24].

Future research on temporal metagenomics and transcriptomic data in 3-dimensional environmental interacting stress factors (temperature, $\mathrm{a}_{w}$ and $\mathrm{CO}_{2}$ ) should provide breakthrough data to decipher the fungal interaction in crops and the dominance of different fungal communities. This would be beneficial in understanding these changes and also identifying beneficial microorganisms, for example potential biocontrol candidates in specific ecological niches for mycotoxin control. These approaches will require parallel development of robust bioinformatics tools to gather data and define patterns in response to interacting environmental stress factors.

\section{Proteomics}

Fungal proteomics research, especially that related to filamentous fungi, has progressed dramatically over the past 10 years. Similar to genomics and transcriptomics, proteomics has evolved to incorporate high-throughput techniques and protocols that allow a faster analysis of large numbers of proteins [25].

In the study of plant pathogenic fungi, the first studies using a proteomic approach were carried out in 1980s with the aim of a better understanding of plant-fungal pathogen interactions through the search of resistance-related proteins [26]. However, although several research studies have used proteomics in order to study the biology and pathogenesis of fungal plant pathogens (e.g., Blumeria graminis f.sp. hordei, Botrytis cinerea, Leptosphaeria maculans, Magnaporthe grisea, Ustilago maydis, Phytophthora infestans), including mycotoxigenic fungi (A. flavus; F. graminearum), the effect of interacting environmental changes on adaptation and toxin production has not been widely considered $[27,28]$.

Lu et al. (2010) compared the secretome of Aspergillus niger when xylose or maltose were supplemented in the medium. Their results showed that although the secretome was strongly affecting the intracellular proteome, this was not significantly changed. Conversely, differences in culture conditions $(\mathrm{pH}$ control versus no $\mathrm{pH}$ control, aeration versus no aeration and stirring versus shaking) had a profound effect on the intracellular proteome [29].

Sørensen et al. (2009) described the effect of the combined addition of lactate and starch in the medium for the growth of $A$. niger. The results showed that fumonisin B2 production was significantly increased. The proteome of $A$. niger was clearly different during growth on media containing 3\% starch, $3 \%$ starch $+3 \%$ lactate or $3 \%$ lactate. The identity of 59 proteins was obtained. Many of these were enzymes involved in primary metabolism and other processes that affect the intracellular level of acetylCoA or NADPH [30]. 
The effect of different light wavelengths on differentially expressed proteins produced by Penicillium verrucosum grown either in the dark or under light with a wavelength of $450 \mathrm{~nm}$ was analysed (Stoll et al., 2014). They identified 46 significantly differential proteins (light vs. dark) comprising proteins of a broad range of isoelectric points and molecular masses. Most proteins were involved in response to stress (e.g. antioxidative proteins, heat shock proteins) and general metabolic processes (e.g. glycolysis, ATP supply). Furthermore, the results indicated that light of short wavelength led to oxidative stress in the fungal cell and under this condition the mycotoxin biosynthesis revealed a shift from ochratoxin A to citrinin [31].

The effect of aw on the proteomic profile of $A$. flavus was investigated by Zhang et al. (2015). A total of 3566 proteins were identified, of which 837 were differentially expressed in response to variations in $\mathrm{a}_{\mathrm{w}}$. Among these, 403 were over-expressed at $0.99 \mathrm{aw}$, whereas 434 were over-expressed at $0.93 \mathrm{aw}$. Two proteins (AFL2G_04330 and KapK) were identified as having a critical role in the induction of aflatoxin biosynthesis [32]. Bai et al. (2015) investigated the changes in transcript and relative protein levels in response to temperature, complementary transcriptomic and proteomic analyses were used to identify changes in $A$. flavus grown at $28^{\circ} \mathrm{C}$ and $37^{\circ} \mathrm{C}$. A total of 3,886 proteins were identified, and 2,832 were reliably quantified. Interestingly the authors pointed out that there was a low correlation between the proteome and transcriptome data, suggesting that post-transcriptional gene regulation may have a very important role on different biological pathways and secondary metabolite gene clusters [33].

\section{Metabolomics}

The fungal metabolome represents the collection of all metabolites in a biological organism (including metabolic intermediates, hormones and other signalling molecules, and secondary metabolites), which are the end products of its gene expression [34]. Metabolomics represents a newer complementary technique to functional genomics as it provides integrative information, i.e., a large number of genes may be involved in the production of one metabolite [35]. This is the reason why the metabolomics approach (both targeted and untargeted) has gained popularity in the field of filamentous fungi, and today these techniques are already being used with different purposes.

Focusing on fungi, the metabolite profile has been used as a common tool in studies of fungal taxonomy and physiology [35] as well as for the early detection of food pathogens and food spoilage microorganisms by means of the volatile production patterns [36]. In addition, metabolomics can be an excellent tool for determining and also predicting the function of unknown genes by comparison with the metabolic perturbations caused by a genetic manipulation such as gene deletion or insertion $[37,38]$. However, the metabolome is also dynamic and is constantly changing in relation with other microorganisms, the host and environmental fluctuations. Luo et al., 2017 [39] recently identified differential metabolites in the interaction zone between two interacting fungi, when compared to those metabolites produced individually in the absence of such interactions. This has provided new information on the molecular ecology of fungal communities on plants. For mycotoxigenic fungi most of the metabolomics studies have been focused on understanding the roles of plant resistance mechanism and pathogen cross-talk at a molecular level [40-43]. It has been demonstrated that the influence of the environment on the synthesis of 
secondary metabolites (SM) by fungi is an important factor to consider [44]. However, few studies have examined targeted SM production patterns in relation to interacting environmental conditions [45-47]. Very recently, Garcia-Cela et al., [45], studied the effect of the environment $\left(10-25^{\circ} \mathrm{C}\right.$ vs $\left.0.90-0.93 \mathrm{aw}\right)$ on targeted metabolite production in natural wheat and wheat inoculated with $F$. graminearum after 15 days storage (Fig. 2). Out of a total of 121 metabolites it was noted that approx. 30-50 metabolites were present at $15-25^{\circ} \mathrm{C}$ regardless of aw conditions. At $10^{\circ} \mathrm{C}$, slower mould colonization occurred resulting in significantly less SMs being produced in both naturally contaminated stored wheat and that inoculated with $F$. graminearum. However, the relative ratio of the compounds produced in significant concentrations in these treatments, were significantly less. Thus, about 20-25 compounds were produced at $>50 \mathrm{ng} / \mathrm{g}$ and $<10$ at $>500 \mathrm{ng} / \mathrm{g}$. This suggests that, surprisingly, there are a wide range of SM compounds which are produced over a range of interacting environmental stress conditions at similar concentrations. More studies are required on other staple food commodities.

Similar experiments were carried out on natural maize and that inoculated with $A$. flavus under different environment conditions $\left(15-35^{\circ} \mathrm{C}\right.$ vs $\left.0.80-0.99 \mathrm{aw}\right)$ after 11 days (Fig. 3). Approx. 20-95 metabolites were present out of total of 168 metabolites analysed. Interestingly, the number of SMs synthetized decreased at $0.95 \mathrm{a}_{\mathrm{w}}$ when comparing 25 and $35^{\circ} \mathrm{C}$ showing a correlation with the environmental conditions. As was observed on wheat, the increase in inoculum of the A. flavus in maize prior to storage promoted the number of SMs produced (Unpublished data).

Only few authors have developed experiments on maize plants looking at interaction between different environmental conditions. Among them, Vaughan et al. 2016 [48] showed how that maize simultaneously exposed to elevated $\mathrm{CO}_{2}$ and drought are even more susceptible to $F$. verticillioides proliferation and also prone to higher levels of fumonisin contamination.

\section{Future trends}

Although the recent improvemements in bioinformatics tools in the last decade the main challenge of systems biology remains the integration of "omics" information to give a more complete picture of living organisms. The contribution of -omics techniques to our better understanding of a range of fungal pathogens including mycotoxigenic species in the last decade is clear. However, under the pressures of changing climate and the food security agenda mycotoxigenic fungi and their mycotoxins may prove to represent increased risks to human and animal health based on the limited data available at the present time. By combining the utilization and integration of holistic and high throughput molecular approaches and appropriate bioinformatics and modelling contributions, significant improvements in the understanding of these fungi will be possible and more appropriate minimisation/control strategies be developed.

Of course, mycotoxigenic fungi do not exist in the field or post-harvest in isolation, but as part of changing ecological communities which are influenced by environmental pressures. These ecological relationships and interactions together with environmental fluxes determines the type and range of secondary metabolites produced. Thus, in the coming years, microbiome approaches studying the microbial communities of food commodities using genomics and transcriptomic approaches will 
help us to better understand mycotoxin production regulation and help to identify the best strategies for the development of better control systems.

The availability of metabolomics techniques to study the production of the full range of secondary metabolites, including toxic ones in food commodities means that a better understanding is necessary of the relationships between these metabolites/toxins and whether their presence individually or in combination represents additive or synergistic impacts on staple foods. Also, we now have better knowledge of the interaction between mycotoxins and their binding by the host plant to for bound or modified mycotoxins. Although there is some data available about the changes in free mycotoxin ratios (Figure 4) we need more data and a better understanding of the impact that changes in environmental stress affect the ratio of free- vs bound mycotoxins, e.g. DON glucosides; FUM related ones etc.). In this area, mycotoxycomic studies involving what perhaps could be called the "mycotoxicome" will produce new valuable information in the coming years.

A better understanding of the relationship between environmental stresses and the production of secondary metabolites at a molecular and transcriptome level may be critical in understand the impact of crop protection approaches in sustainable food production systems. In addition, we need more knowledge of the potential for a switching of ratios of mycotoxins produced by the same toxigenic species (e.g. $A$. flavus: aflatoxins vs cyclopiazonic acid) and whether other secondary metabolites may become more important under environmental stresses such as climate change factors. Thus new-omics based approaches are essential for a better understanding of the conditions under which mycotoxin contamination of staple foods can be minimised and identify strategies which will be beneficial in terms of food safety and food security.

\section{References}

1. Medina A, Akbar A, Baazeem A, Rodriguez A, Magan N: Climate change, food security and mycotoxins: Do we know enough? Fungal Biol Rev 2017, 31.

The authors review the current state of research in relation to gene expression of important genes in different mycotoxin production pathways in different fungi. They review the environmental changes that are forecasted in the coming decades and the potential impact on mycotoxin contamination of staple foods

2. Medina Á, González-Jartín JM, Sainz MJ: Impact of global warming on mycotoxins. Curr Opin Food Sci 2017, 18:76-81.

3. Botana LM, Sainz MJ: Climate change and mycotoxins. De Gruyter; 2015.

4. Magan N, Aldred D: Chapter 2 Environmental fluxes and fungal interactions: Maintaining a competitive edge. Br Mycol Soc Symp Ser 2008, 27:19-35.

5. Bhatnagar D, Rajasekaran K, Payne G, Brown R, Yu J, Cleveland T: The "omics" tools: genomics, proteomics, metabolomics and their potential for solving the aflatoxin contamination problem. World Mycotoxin J 2008, $1: 3-12$.

This is an important publication that set the scene on the next developments for the use of -omics techniques to study Aspergillus flavus and its production of aflatoxins. 
6. Bhatnagar D, Rajasekaran K, Gilbert M, Cary JW, Magan N: Advances in molecular and genomic research to safeguard food and feed supply from aflatoxin contamination. World Mycotoxin J 2018, 11:47-72.

7. Medina A, Schmidt-Heydt M, Rodríguez A, Parra R, Geisen R, Magan N: Impacts of environmental stress on growth, secondary metabolite biosynthetic gene clusters and metabolite production of xerotolerant/xerophilic fungi. Curr Genet 2015, 61.

8. Magan N, Medina A: Mycotoxins, food security and climate change: Do we know enough? Microbiol Today 2016, 43.

9. Medina Á, Rodríguez A, Magan N: Climate change and mycotoxigenic fungi: Impacts on mycotoxin production. Curr Opin Food Sci 2015, 5.

10. Medina A, Gilbert MK, Mack BM, OBrian GR, Rodríguez A, Bhatnagar D, Payne $\mathrm{G}$, Magan N: Interactions between water activity and temperature on the Aspergillus flavus transcriptome and aflatoxin $\mathrm{B}_{1}$ production. Int $J$ Food Microbiol 2017, 256.

11. Chakrabortti A, Li J, Liang Z-X: Complete Genome Sequence of the Filamentous Fungus Aspergillus westerdijkiae Reveals the Putative Biosynthetic Gene Cluster of Ochratoxin A. Genome Announc 2016, 4:e00982-16.

12. Satterlee T, Cary JW, Calvo AM: RmtA, a Putative Arginine Methyltransferase, Regulates Secondary Metabolism and Development in Aspergillus flavus. PLoS One 2016, 11:e0155575.

13. Yang K, Liang L, Ran F, Liu Y, Li Z, Lan H, Gao P, Zhuang Z, Zhang F, Nie X, et al.: The DmtA methyltransferase contributes to Aspergillus flavus conidiation, sclerotial production, aflatoxin biosynthesis and virulence. Sci Rep 2016, 6:23259.

14. Visentin I, Montis V, Döll K, Alabouvette C, Tamietti G, Karlovsky P, Cardinale F: Transcription of Genes in the Biosynthetic Pathway for Fumonisin Mycotoxins Is Epigenetically and Differentially Regulated in the Fungal Maize Pathogen Fusarium verticillioides. Eukaryot Cell 2012, 11:252-259.

15. Abdel-Hadi A, Carter D, Magan N: Temporal monitoring of the nor-1 (afID) gene of Aspergillus flavus in relation to aflatoxin $B_{1}$ production during storage of peanuts under different water activity levels. $J$ Appl Microbiol 2010, 109:1914-1922.

16. Samsudin NIP, Rodriguez A, Medina A, Magan N: Efficacy of fungal and bacterial antagonists for controlling growth, FUM1 gene expression and fumonisin B1 production by Fusarium verticillioides on maize cobs of different ripening stages. Int J Food Microbiol 2017, 246:72-79.

17. Geisen R, Schmidt-Heydt M, Stoll D, Touhami N: Aspects of the Occurrence, Genetics, and Regulation of Biosynthesis of the Three Food Relevant Penicillium Mycotoxins: Ochratoxin A, Citrinin, and Patulin. In Physiology and Genetics. . Springer International Publishing; 2018:413-433.

18. Yu J, Fedorova ND, Montalbano BG, Bhatnagar D, Cleveland TE, Bennett JW, Nierman WC: Tight control of mycotoxin biosynthesis gene expression in Aspergillus flavus by temperature as revealed by RNA-Seq. FEMS Microbiol Lett 2011, 322:145-149.

The authors describe here for the first time the very close control that temperature has controlling gene expression using RNA-seq. They also demonstrated this control has impact on the aflatoxin production pathway. 
19. Medina A, Gilbert MK, Mack BM, OBrian GR, Rodríguez A, Bhatnagar D, Payne $G$, Magan N: Interactions between water activity and temperature on the Aspergillus flavus transcriptome and aflatoxin $\mathrm{B}_{1}$ production. Int $\mathrm{J}$ Food Microbiol 2017, 256:36-44.

This is the first paper where the dual interaction between tempareture and water activity on the gene expression of Aspergillus flavus growing in irradiated maize kernels was evaluated by using RNA-seq. It was demonstrated that both have a very important effect on key genes in the aflatoxin production pathway.

20. Gilbert M, Medina A, Mack B, Lebar M, Rodríguez A, Bhatnagar D, Magan N, Obrian G, Payne G: Carbon Dioxide Mediates the Response to Temperature and Water Activity Levels in Aspergillus flavus during Infection of Maize Kernels. Toxins (Basel) 2017, 10:5.

The authors study the gene expression of A.flavus growing in irradiated maize kernels at different temperatures, water activities and atmospheric $\mathrm{CO}_{2}$ concentrations. They found that the three way interactions, including $\mathrm{CO}_{2}$, have an impact in the gene expression of important secondary metabolite genes including tjose in the the aflatoxin production pathway.

21. Hertz M, Jensen IR, Jensen L $\varnothing$, Thomsen SN, Winde J, Dueholm MS, Sørensen LH, Wollenberg RD, Sørensen HO, Sondergaard TE, et al.: The fungal community changes over time in developing wheat heads. Int $\mathrm{J}$ Food Microbiol 2016, 222:30-39.

22. Walder F, Schlaeppi K, Wittwer R, Held AY, Vogelgsang S, van der Heijden MGA: Community Profiling of Fusarium in Combination with Other PlantAssociated Fungi in Different Crop Species Using SMRT Sequencing. Front Plant Sci 2017, 8:2019.

23. Gdanetz K, Trail F: The Wheat Microbiome Under Four Management Strategies, and Potential for Endophytes in Disease Protection. Phytobiomes 2017, 1:158-168.

24. Xing F, Ding N, Liu X, Selvaraj JN, Wang L, Zhou L, Zhao Y, Wang Y, Liu Y: Variation in fungal microbiome (mycobiome) and aflatoxins during simulated storage of in-shell peanuts and peanut kernels. Sci Rep 2016, 6:25930.

25. Gonzalez-Fernandez R, Jorrin-Novo J V.: Contribution of Proteomics to the Study of Plant Pathogenic Fungi. J Proteome Res 2012, 11:3-16.

26. Schottens-Toma IMJ, de Wit PJGM: Purification and primary structure of a necrosis-inducing peptide from the apoplastic fluids of tomato infected with Cladosporium fulvum (syn. Fulvia fulva). Physiol Mol Plant Pathol 1988, 33:59-67.

27. Crespo-Sempere A, Gil JV, Martínez-Culebras PV: Proteome analysis of the fungus Aspergillus carbonarius under ochratoxin A producing conditions. Int J Food Microbiol 2011, 147:162-169.

28. Nöbauer K, Hummel K, Mayrhofer C, Ahrens M, Setyabudi FMC, SchmidtHeydt M, Eisenacher M, Razzazi-Fazeli E: Comprehensive proteomic analysis of Penicillium verrucosum. Proteomics 2017, 17:1600467.

29. Lu X, Sun J, Nimtz M, Wissing J, Zeng A-P, Rinas U: The intra- and extracellular proteome of Aspergillus niger growing on defined medium with xylose or maltose as carbon substrate. Microb Cell Fact 2010, 9:23.

30. Sørensen LM, Lametsch R, Andersen MR, Nielsen P V, Frisvad JC: Proteome 
analysis of Aspergillus niger: Lactate added in starch-containing medium can increase production of the mycotoxin fumonisin B2 by modifying acetyl-CoA metabolism. BMC Microbiol 2009, 9:255.

31. Stoll DA, Link S, Kulling S, Geisen R, Schmidt-Heydt M: Comparative proteome analysis of Penicillium verrucosum grown under light of short wavelength shows an induction of stress-related proteins associated with modified mycotoxin biosynthesis. Int J Food Microbiol 2014, 175:2029.

32. Zhang F, Zhong H, Han X, Guo Z, Yang W, Liu Y, Yang K, Zhuang Z, Wang S: Proteomic profile of Aspergillus flavus in response to water activity. Fungal Biol 2015, 119:114-124.

33. Bai Y, Wang S, Zhong H, Yang Q, Zhang F, Zhuang Z, Yuan J, Nie X, Wang $S$ : Integrative analyses reveal transcriptome-proteome correlation in biological pathways and secondary metabolism clusters in $A$. flavus in response to temperature. Sci Rep 2015, 5:14582.

34. Fiehn O: Combining genomics, metabolome analysis, and biochemical modelling to understand metabolic networks. Comp Funct Genomics 2001, 2:155-68.

35. Smedsgaard J, Nielsen J: Metabolite profiling of fungi and yeast: from phenotype to metabolome by MS and informatics. J Exp Bot 2005, 56:273286.

36. Pinu FR: Early detection of food pathogens and food spoilage microorganisms: Application of metabolomics. Trends Food Sci Technol 2016, 54:213-215.

37. Bhatnagar D, Rajasekaran K, Payne G, Brown R, Yu J, Cleveland T: The "omics" tools: genomics, proteomics, metabolomics and their potential for solving the aflatoxin contamination problem. World Mycotoxin J 2008, 1:3-12.

38. Weckwerth W: METABOLOMICS IN SYSTEMS BIOLOGY. Annu Rev Plant Biol 2003, 54:669-689.

39. Luo F, Zhong Z, Liu L, Igarashi Y, Xie D, Li N: Metabolomic differential analysis of interspecific interactions among white rot fungi Trametes versicolor, Dichomitus squalens and Pleurotus ostreatus. Sci Rep 2017, 7:5265.

40. Nathanail A V., Varga E, Meng-Reiterer J, Bueschl C, Michlmayr H, Malachova A, Fruhmann P, Jestoi M, Peltonen K, Adam G, et al.: Metabolism of the Fusarium Mycotoxins T-2 Toxin and HT-2 Toxin in Wheat. J Agric Food Chem 2015, 63:7862-7872.

41. Gauthier L, Atanasova-Penichon V, Chéreau S, Richard-Forget F:

Metabolomics to Decipher the Chemical Defense of Cereals against Fusarium graminearum and Deoxynivalenol Accumulation. Int $\mathrm{J} \mathrm{Mol} \mathrm{Sci}$ 2015, 16:24839-24872.

42. Scala V, Camera E, Ludovici M, Dall'Asta C, Cirlini M, Giorni P, Battilani P, Bello $C$, Fabbri AA, Fanelli $C$, et al.: Fusarium verticillioides and maize interaction in vitro : relationship between oxylipin cross-talk and fumonisin synthesis. World Mycotoxin J 2013, 6:343-351.

43. Warth B, Parich A, Bueschl C, Schoefbeck D, Neumann NKN, Kluger B, Schuster K, Krska R, Adam G, Lemmens M, et al.: GC-MS based targeted metabolic profiling identifies changes in the wheat metabolome following deoxynivalenol treatment. Metabolomics 2015, 11:722-738. 
44. Hautbergue T, Jamin EL, Debrauwer L, Puel O, Oswald IP: From genomics to metabolomics, moving toward an integrated strategy for the discovery of fungal secondary metabolites. Nat Prod Rep 2018, 35:147-173.

45. Garcia-Cela E, Kiaitsi E, Medina A, Sulyok M, Krska R, Magan N: Interacting environmental stress factors affects targeted metabolomic profiles in stored natural wheat and that inoculated with $\boldsymbol{F}$. graminearum. Toxins (Basel) 2018, 10.

46. Mogensen JM, Sørensen SM, Sulyok M, van der Westhuizen L, Shephard GS, Frisvad JC, Thrane U, Krska R, Nielsen KF: Single-kernel analysis of fumonisins and other fungal metabolites in maize from South African subsistence farmers. Food Addit Contam Part A 2011, 28:1-11.

47. Adetunji M, Atanda O, Ezekiel CN, Sulyok M, Warth B, Beltrán E, Krska R, Obadina O, Bakare A, Chilaka CA: Fungal and bacterial metabolites of stored maize (Zea mays, L.) from five agro-ecological zones of Nigeria. Mycotoxin Res 2014, 30:89-102.

48. Vaughan MM, Huffaker A, Schmelz EA, Dafoe NJ, Christensen SA, McAuslane HJ, Alborn HT, Allen LH, Teal PEA: Interactive Effects of Elevated $\mathrm{CO}_{2}$ and Drought on the Maize Phytochemical Defense Response against Mycotoxigenic Fusarium verticillioides. PLoS One 2016, 11:e0159270. 
Mycotoxin Production

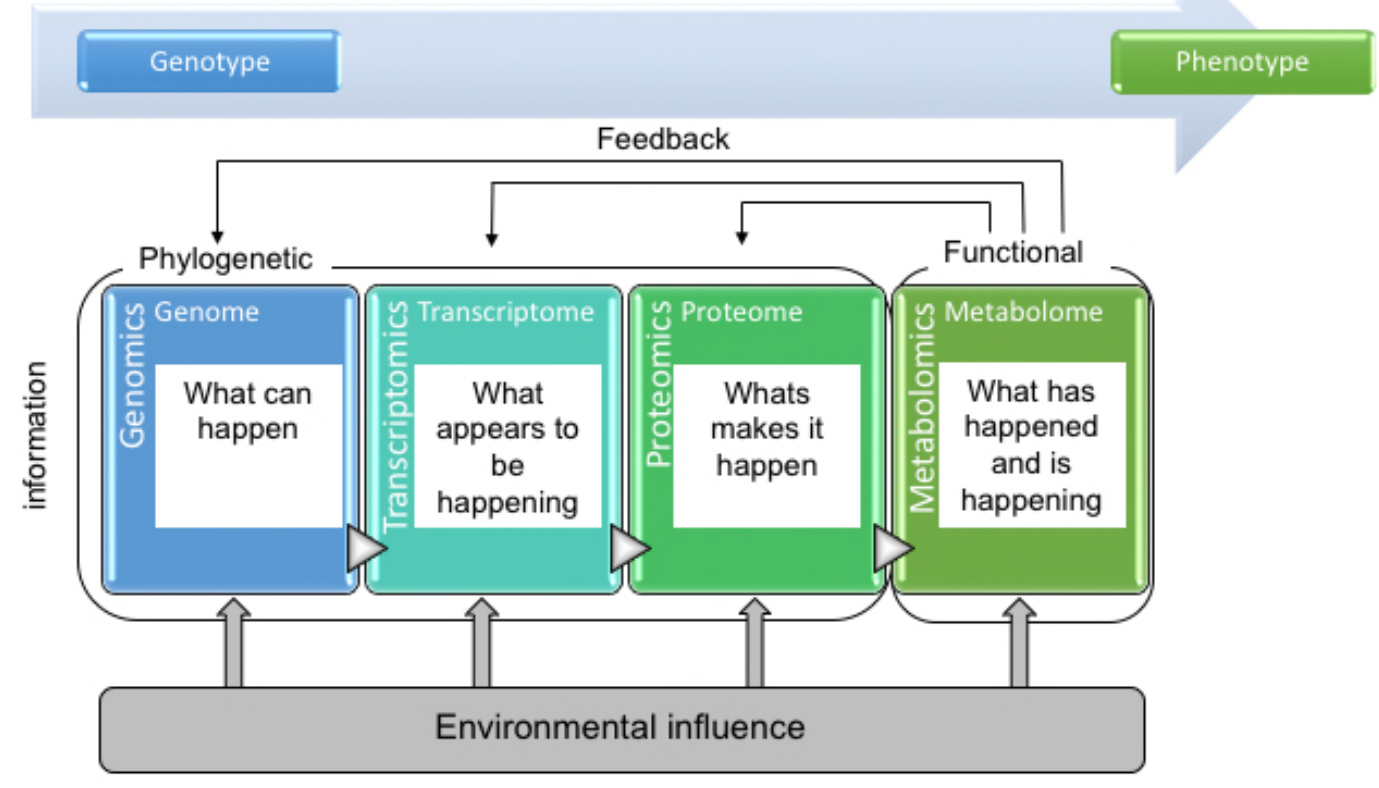

Figure 1

Figure 1. Relationship between mycotoxin production and the relationship between the diferent-OMIC techniques. 


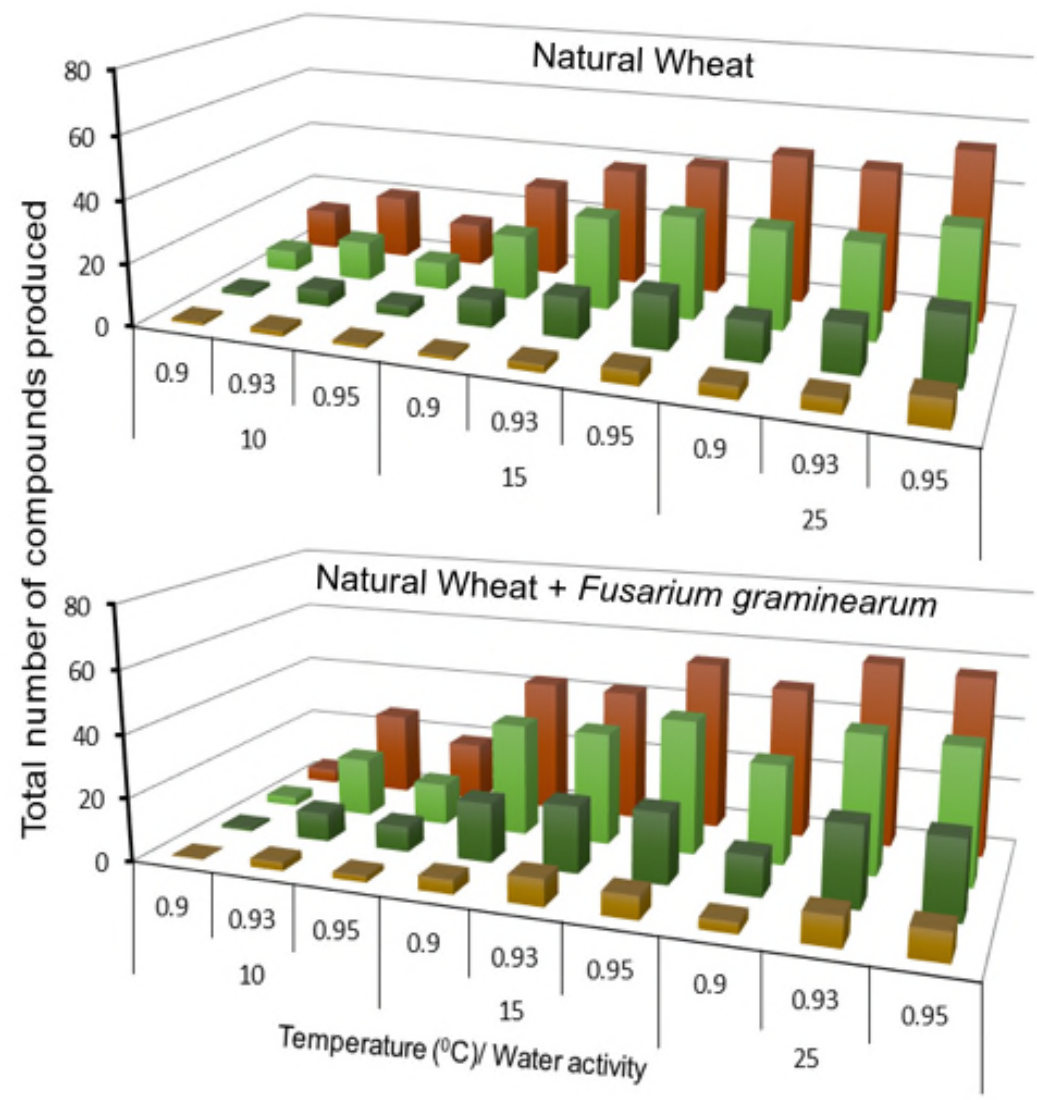

- Number compunds higher than $500 \mathrm{ng} / \mathrm{g}$

- Number compunds higher than $50 \mathrm{ng} / \mathrm{g}$

= Number compunds higher than $5 \mathrm{ng} / \mathrm{g}$

- Number of compounds

- Number compunds higher than $500 \mathrm{ng} / \mathrm{g}$

- Number compunds

higher than $50 \mathrm{ng} / \mathrm{g}$

= Number compunds

higher than $5 \mathrm{ng} / \mathrm{g}$

- Number of compounds

Figure 2. Comparison of the number and amount of metabolites produced between natural wheat and natural wheat contaminated with Fusarium graminearum and incubated under different environmental conditions 


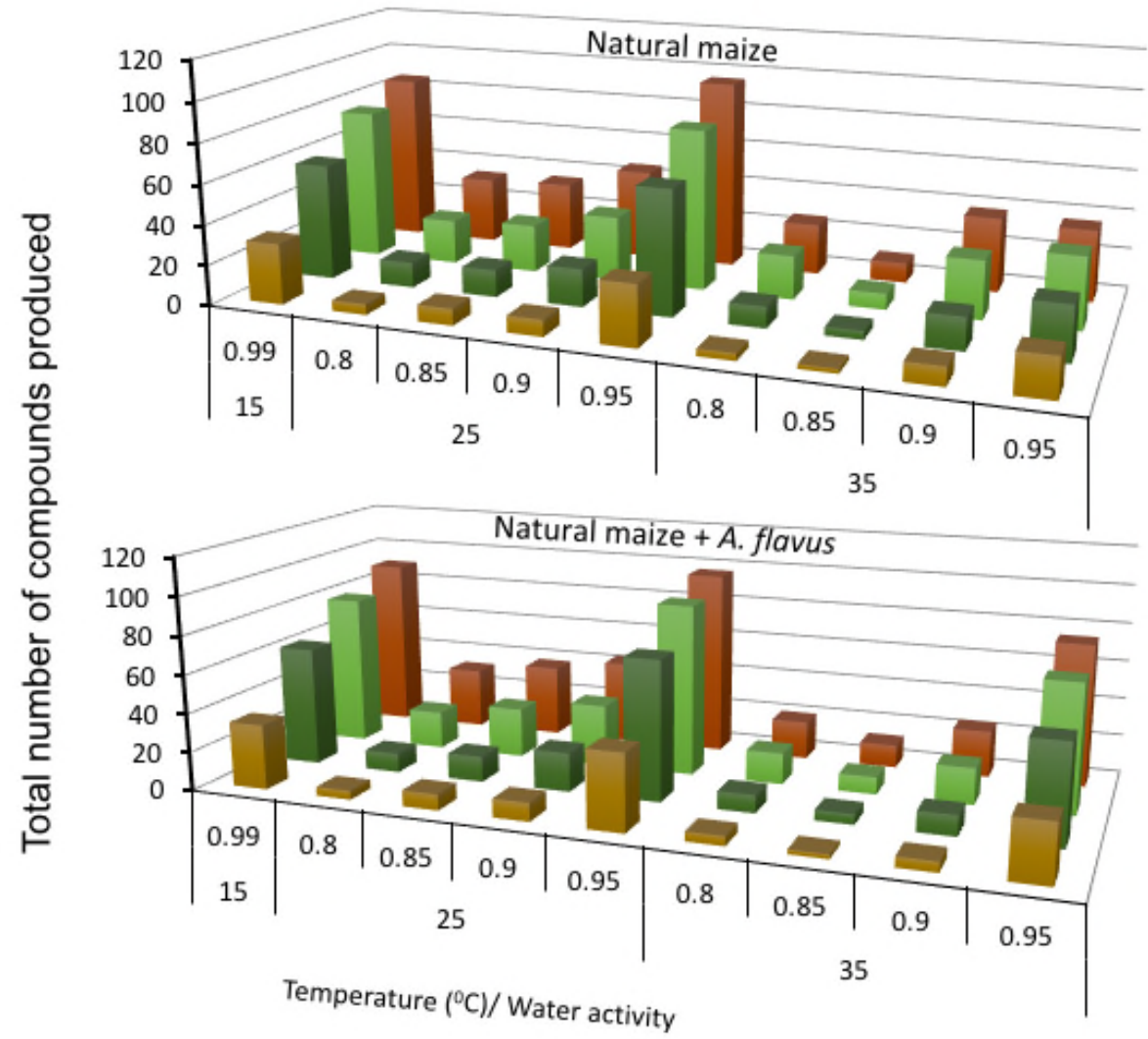

n Number compunds higher than $500 \mathrm{ng} / \mathrm{g}$

- Number compunds

higher than $50 \mathrm{ng} / \mathrm{g}$

n Number compunds

higher than $5 \mathrm{ng} / \mathrm{g}$

- Number of compounds

- Number compunds higher than $500 \mathrm{ng} / \mathrm{g}$

= Number compunds

higher than $50 \mathrm{ng} / \mathrm{g}$

= Number compunds

higher than $5 \mathrm{ng} / \mathrm{g}$

- Number of compounds

Figure 3. Comparison of the number and amount of metabolites produced between natural maize and natural mize contaminated with Aspergillus flavus and incubated under different environmental conditions 


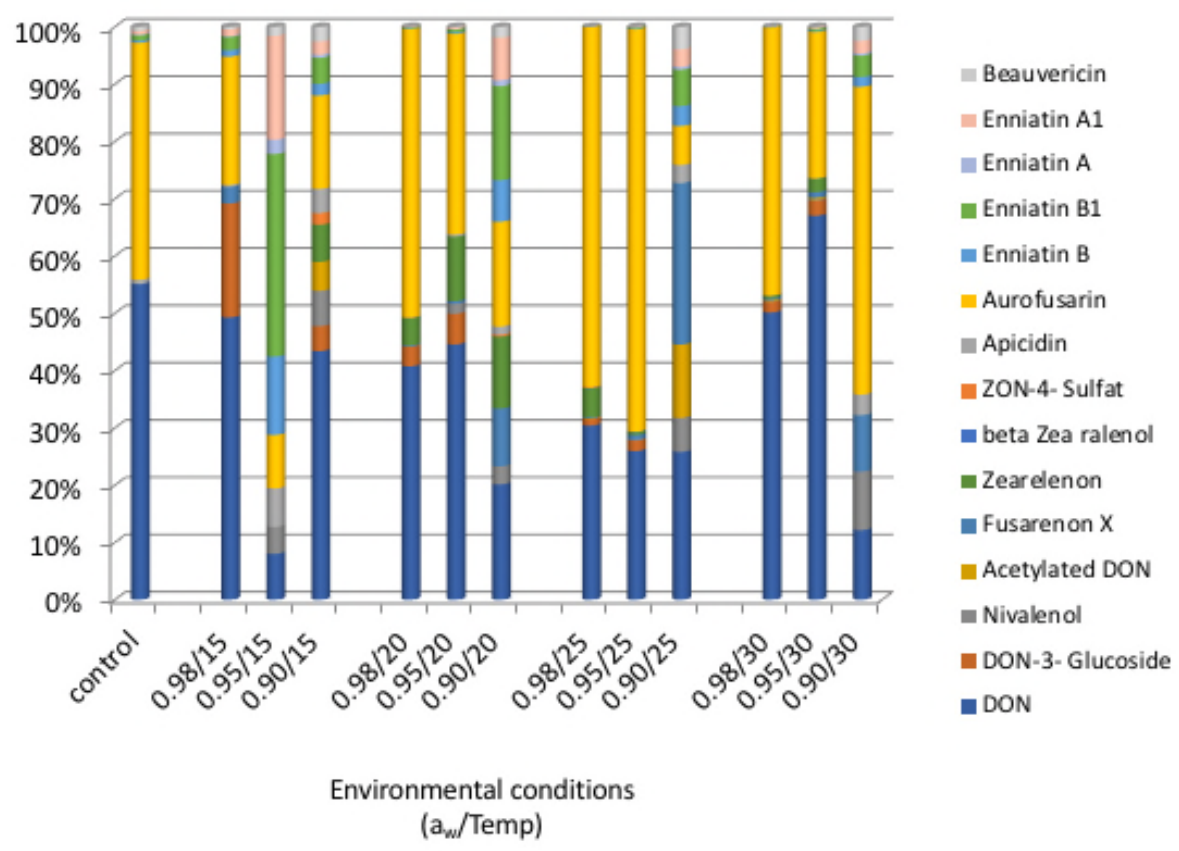

Figure 4 Relative concentrations of different mycotoxins found in natural wheat grain inoculated with F.graminearum and stored under different conditions for 25 days 